\title{
Editorial Introduction to ACM THRI Volume 8, Issue 2
}

\author{
ODEST CHADWICKE JENKINS, University of Michigan, USA \\ SELMA ŠABANOVIĆ, Indiana University Bloomington, USA
}

Our second issue of ACM THRI this year brings together another collection of insightful and thoughtful articles pushing the boundaries of thought in Human-Robot Interaction. Furthering ideas at the meeting of HRI and artificial intelligence, our first article, from Heard et al., presents a human workload assessment algorithm to enable dynamic task allocation that improves the collective performance of human-robot teams. Stefanov et al. use artificial neural networks for the generation of robot gaze directions in dialogues including multiple individuals that analyze human behavior across combinations of movement and speech signals.

The middle section of this issue focuses on the perspectives of older adults and their acceptance of robotic technology. Yeh et al. deliver findings that that can improve acceptance of robots through establishing personal relevance, even though such populations tend to have more negative initial reactions to robots. Jung and Ludden explored the potential for robotic exoskeletons to be accepted by older adults as viable mobility aids, yielding insights that will be important for the design of usable devices with this technology.

The final two articles of this issue address problems in characterizing expressiveness in humanrobot systems. St-Onge et al. present a neural network model to capture features of expressiveness from wearable human sensors to produce expressive swarm behavior for teams of mobile robots. Schulz et al. survey existing literature in social robotics with respect to unifying principles of expressive movement in animation.

Our editorial board remains energized and enthusiastic by the quality and diversity of works submitted to ACM THRI. The articles above are a small sampling of the many compelling ideas we have the privilege to review, discuss, provide feedback to, and publish. Continuing with the publication of such articles in our second year, ACM THRI is on track to realize its commitment to intellectual impact, sustainability, and inclusion for the field of Human-Robot Interaction. We invite our readers to contribute to our progress for the field of HRI with their own submissions and timely response to review requests.

(C) 2019 Copyright held by the owner/author(s).

2573-9522/2019/05-ART6

https://doi.org/10.1145/3328224 